\title{
Partial Fraction Evaluation and Incomplete Decomposition of a Rational Function Whose Denominator Contains a Repeated Polynomial Factor
}

\author{
By J. F. Mahoney \\ Dedicated to Peter Henrici
}

\begin{abstract}
Attention is directed to those proper rational functions whose denominators may be expressed as the product of an $N$ th degree polynomial raised to the $K$ th power and another polynomial of degree $M$. A method is presented for decomposing such a rational function into the sum of the $K$ partial fraction terms which proceed from the repeated polynomial plus a proper rational function which completes the equality. Use is made of an extended version of Horner's scheme. Two numerical examples and an operations count are presented. The method is free of complex arithmetic provided that all of the coefficients of the entering polynomials are real.
\end{abstract}

Introduction. Consider the proper rational function

$$
F(s)=\frac{B(s)}{Q^{K}(s) A(s)},
$$

where $Q(s)$ is a polynomial of degree $N(N \geqslant 1), A(s)$ is a polynomial of degree $M$ which shares none of its zeros with $Q(s) ; B(s)$ is a polynomial of degree $m$ $(m<N K+M)$; and $K$ is an integer greater than zero. An efficient way of finding $E(s)$ and the $C_{j}(s)$ in the expression

$$
F(s)=\sum_{j=1}^{K} \frac{C_{j}(s)}{Q^{K-j+1}(s)}+\frac{E(s)}{A(s)}
$$

is now presented, where $E(s)$ is a polynomial of degree less than $M$, and the $C_{j}(s)$ are polynomials of degrees less than $N$. Henrici discusses many important aspects of the problem. When $N=1$, one may use his Method B [1, p. 555] to solve for the $C_{j}(s)$, which in this case are constants. His Method C [1, p. 556], [2], may be used in the case where $K=1$. Actually, this method may be used when $K>1$ by writing $Q^{K}(s)$ as $\tilde{Q}(s)$, which has the effect of reducing the problem to the $K=1$ case. For this problem one may find $\tilde{C}_{1}(s)$ which, in turn, by repeated use of long division, may be written as $C_{1}(s)+C_{2}(s) Q(s)+\cdots+C_{K}(s) Q^{K-1}(s)$. Subsequent division by $Q^{K}(s)$ gives the summation portion of (2).

Received March 8, 1983; revised November 16, 1983 and February 10, 1984.

1980 Mathematics Subject Classification. Primary 65F99. 
The present work enlarges the foregoing to cover the cases where both $K$ and $N$ are greater than or equal to unity. When $N=2$, and $K>1$, we have the commonly occurring partial fractions problem associated with a repeated quadratic factor in the denominator of $F(s)$. There appears to be little demand for handling the case of $N>2$ and $K>1$, but this might be because previously no good methodology existed.

An appealing aspect of the subject method is that the algebra encountered is entirely real, given that the coefficients of $A(s), B(s)$, and $Q(s)$ are also real.

Expansion of an Arbitrary Polynomial in Powers of $Q(s)$. Central to the development is the ability to rewrite the polynomials $A(s)$ and $B(s)$ as power series in $Q(s)$ where the coefficients of the power series are themselves polynomials whose degrees are all less than $N$.

Let $Q(s)$ be written monically as

$$
Q(s)=s^{N}-\sum_{i=1}^{N} q_{i} s^{i-1},
$$

and consider polynomials of the form

$$
A_{i}(s)=\sum_{j=0}^{M-i N} a_{i j} s^{j}, \quad i=0,1, \ldots, u-1,
$$

where $A(s)$ is denoted by $A_{0}(s)$. Owing to the division process $A_{i}(s) / Q(s)$ one may write

$$
A_{i}(s)=\alpha_{i+1}(s)+A_{i+1}(s) Q(s), \quad i=0,1, \ldots, u-1,
$$

where $A_{u}(s)=0$, and hence $\alpha_{u}(s)=A_{u-1}(s)$. The $\alpha_{i+1}(s)$ are the "remainder" polynomials of degrees less than $N$; the $A_{i+1}(s)$ are the "quotient" polynomials; and $u$ is the integer given by

$$
\frac{M+1}{N} \leqslant u \leqslant \frac{M+N}{N}
$$

Combination of the $u$ renditions of (5) gives

$$
A(s)=\sum_{i=1}^{u} \alpha_{i}(s) Q^{i-1}(s) .
$$

The $\alpha_{i}(s)$ may be written as

$$
\alpha_{i}(s)=\sum_{j=1}^{N} \alpha_{i j} s^{j-1}, \quad i=1,2, \ldots, u .
$$

The $\alpha_{i}(s)$ which appear in (7) may be found by performing the appropriate long divisions. When $Q(s)$ is linear $(N=1)$, the $\alpha_{i}(s)$ are numbers and may be found by Horner's scheme. When $Q(s)$ is not linear $(N>1)$, there is a straightforward generalization of Horner's scheme which may be used.

Figure 1 serves to illustrate one step of the process of finding $\alpha_{i+1}(s)$ and $A_{i+1}(s)$ when $A_{i}(s)$ is given. The aim is to be able to write $A_{i}(s)$ according to (5). The coefficients of $A_{i}(s)$ are placed at the top of the tableau. The next $N$ rows are for "bookkeeping." The last row shown is obtained by addition of the $N+1$ rows above, and is segregated into two portions: the left-hand portion contains the 
coefficients of $A_{i+1}(s)$, while the right-hand portion contains the coefficients of $\alpha_{i+1}(s)$. Multiplication of the bracket which contains the coefficients of $A_{i+1}(s)$ by $q_{k}, 1 \leqslant k \leqslant N$, followed by a shift to the right of $N-k+1$ places results in the formation of the $k$ th bookkeeping row.

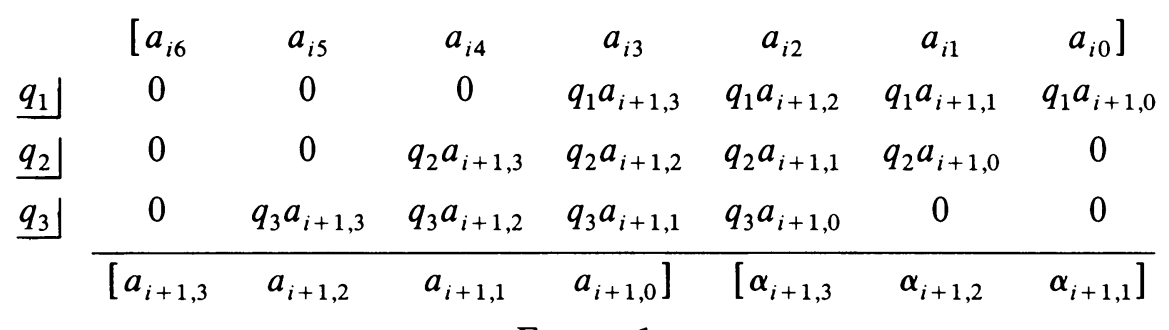

FIGURE 1

A portion of the extended version of Horner's scheme for the case of $M-i N=6$, and $N=3$.

The entire process starts with $i=0$, so that the coefficients of $A(s)$ form the top row of the tableau. The first step, with $i=0$, yields the coefficients of $\alpha_{1}(s)$ and $A_{1}(s)$. Through successive incrementation of $i$, one gains the coefficients of $\alpha_{2}(s), \ldots, \alpha_{u}(s)$, where, in particular, $\alpha_{u}(s)=A_{u-1}(s)$.

By means of the decomposition method just outlined, one may also write

$$
B(s)=\sum_{i=1}^{v} \beta_{i}(s) Q^{i-1}(s),
$$

where the $\beta_{i}(s)$ are polynomials of degrees less than $N$, and $v$ is the integer given by

$$
\frac{m+1}{N} \leqslant v \leqslant \frac{m+N}{N} .
$$

Since $v \leqslant u+K$ we are also led to

$$
B(s)=\sum_{k=1}^{u+K} \beta_{k}(s) Q^{k-1}(s),
$$

where any $\beta_{k}(s)$ for which $k>v$ is taken to be zero. Likewise,

$$
E(s)=\sum_{k=1}^{u} \varepsilon_{k}(s) Q^{k-1}(s)
$$

where the $\varepsilon_{k}(s)$ are polynomials whose degrees are less than $N$.

Development of the Algorithm. Combination of (1), (2), and (7) yields

$$
B(s)=\left[\sum_{i=1}^{u} \alpha_{i}(s) Q^{i-1}(s)\right]\left[\sum_{j=1}^{K} C_{j}(s) Q^{j-1}(s)\right]+E(s) Q^{K}(s) .
$$

By the decomposition process outlined, one may write

$$
\alpha_{i}(s) C_{j}(s)=X_{i j}(s)+Y_{i j}(s) Q(s), \quad 1 \leqslant i \leqslant u, 1 \leqslant j \leqslant K,
$$

where the $X_{i j}(s)$ are polynomials whose degrees may be as great as $N-1$, while the $Y_{i j}(s)$ are polynomials whose degrees are $N-2$ at most. Both $X_{i j}(s)$ and $Y_{i j}(s)$ are defined to be zero if either of their indices breach the bounds stated in (14). 
Insertion of (11), (12), and (14) into (13) gives, after rearrangement,

$$
\begin{aligned}
& \sum_{k=1}^{u+K} \beta_{k}(s) Q^{k-1}(s) \\
& \quad=\sum_{k=1}^{u+K} Q^{k-1}(s)\left[\varepsilon_{k-K}(s)+\sum_{j=1}^{k}\left(X_{k-j+1, j}(s)+Y_{k-j, j}(s)\right)\right]
\end{aligned}
$$

provided $\varepsilon_{k-K}(s)$ is taken to be zero whenever $k-K$ is not positive. Since (15) is an identity, the leading summation signs may be dropped yielding

$$
\varepsilon_{k-K}(s)+\sum_{j=1}^{k}\left(X_{k-j+1, j}(s)+Y_{k-j, j}(s)\right)=\beta_{k}(s), \quad k=1,2, \ldots, u+K .
$$

This equation is also valid when the polynomials are replaced by the corresponding $N$ component column vectors whose entries are the coefficients of the respective polynomials. Define

$$
\beta_{i}=\left[\beta_{i 1}, \beta_{i 2}, \ldots, \beta_{i N}\right]^{T}, \quad \varepsilon_{i}=\left[\varepsilon_{i 1}, \varepsilon_{i 2}, \ldots, \varepsilon_{i N}\right]^{T}
$$

to be the vectors which correspond to $\beta_{i}(s)$ and $\varepsilon_{i}(s)$, respectively. Here, for example, $\beta_{i j}, j=1,2, \ldots, N$, is the coefficient of $s^{j-1}$ in $\beta_{i}(s)$. Similarly, the vectors which replace $X_{i j}(s)$ and $Y_{i j}(s)$ are, respectively,

$$
\mathbf{x}_{i j}=\left[x_{i j 1}, x_{i j 2}, \ldots, x_{i j N}\right]^{T}, \quad \mathbf{y}_{i j}=\left[y_{i j 1}, y_{i j 2}, \ldots, y_{i j N}\right]^{T} .
$$

Substitution into (16) gives

$$
\boldsymbol{\varepsilon}_{k-K}+\sum_{j=1}^{k}\left(\mathbf{x}_{k-j+1, j}+\mathbf{y}_{k-j, j}\right)=\boldsymbol{\beta}_{k}, \quad k=1,2, \ldots, u+K .
$$

We need to express $\mathbf{x}_{i j}$ and $\mathbf{y}_{i j}$ in terms of the unknown vectors $\mathbf{c}_{j}$ whose elements are the coefficients of $C_{j}(s)$, where

$$
C_{j}(s)=\sum_{i=1}^{N} c_{j i} s^{i-1}, \quad \mathbf{c}_{j}=\left[c_{j 1}, c_{j 2}, \ldots, c_{j N}\right]^{T} .
$$

Comparison of the coefficients of $s^{0}, s^{1}, \ldots, s^{N-1}$ of (14) leads to the relation

$$
\mathbf{S}_{i} \mathbf{c}_{j}=\mathbf{x}_{i j}-\mathbf{V y}_{i j} \text {, }
$$

while comparison of the coefficients of $s^{N}, s^{N+1}, \ldots, s^{2 N-1}$ gives

$$
\mathbf{U}_{i} \mathbf{c}_{j}=\mathbf{W} \mathbf{y}_{i j} \text {, }
$$

where
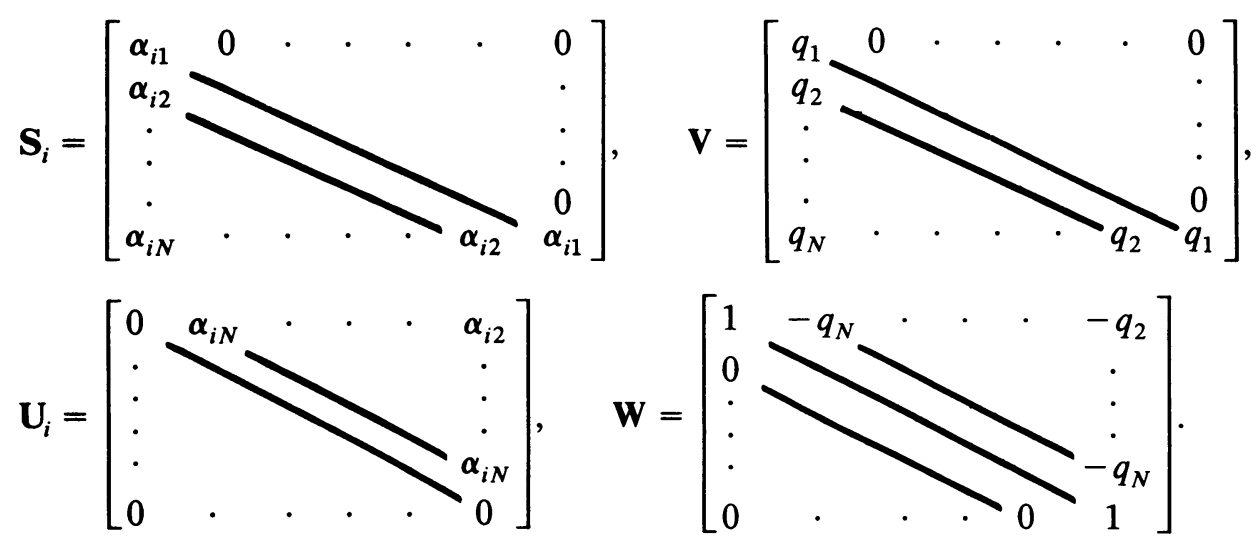
Since $\mathrm{W}^{-1}$ surely exists, (18) and (19) may be written as

$$
\mathbf{y}_{i j}=\mathbf{W}^{-1} \mathbf{U}_{i} \mathbf{c}_{j}
$$

and

$$
\mathbf{x}_{i j}=\left[\mathbf{S}_{i}+\mathbf{V} \mathbf{W}^{-1} \mathbf{U}_{i}\right] \mathbf{c}_{j} .
$$

Insertion of (20) and (21) into (17) gives

$$
\begin{aligned}
\boldsymbol{\varepsilon}_{k-K}+\sum_{j=1}^{k}\left[\mathbf{S}_{k-j+1}+\mathbf{V} \mathbf{W}^{-1} \mathbf{U}_{k-j+1}+\mathbf{W}^{-1} \mathbf{U}_{k-j}\right] \mathbf{c}_{j} & =\boldsymbol{\beta}_{k}, \\
k & =1,2, \ldots, u+K .
\end{aligned}
$$

Define

$$
\mathbf{T}_{i}=\mathbf{S}_{i}+\mathbf{V W}^{-1} \mathbf{U}_{i}+\mathbf{W}^{-1} \mathbf{U}_{i-1}, \quad i=1,2, \ldots, u+1,
$$

where, in particular,

$$
\mathbf{T}_{1}=\mathbf{S}_{1}+\mathbf{V W}^{-1} \mathbf{U}_{1}, \quad \mathbf{T}_{u+1}=\mathbf{W}^{-1} \mathbf{U}_{u} .
$$

We may now write (22) as

$$
\boldsymbol{\varepsilon}_{k-K}+\sum_{j=1}^{k} \mathbf{T}_{k-j+1} \mathbf{c}_{j}=\boldsymbol{\beta}_{k}, \quad k=1,2, \ldots, u+K .
$$

The first $K$ of these equations may be rendered as

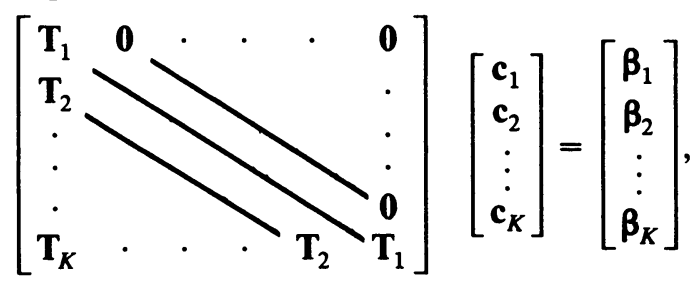

from which the $c_{j}$ may be found. The remaining $u$ equations of (24) become

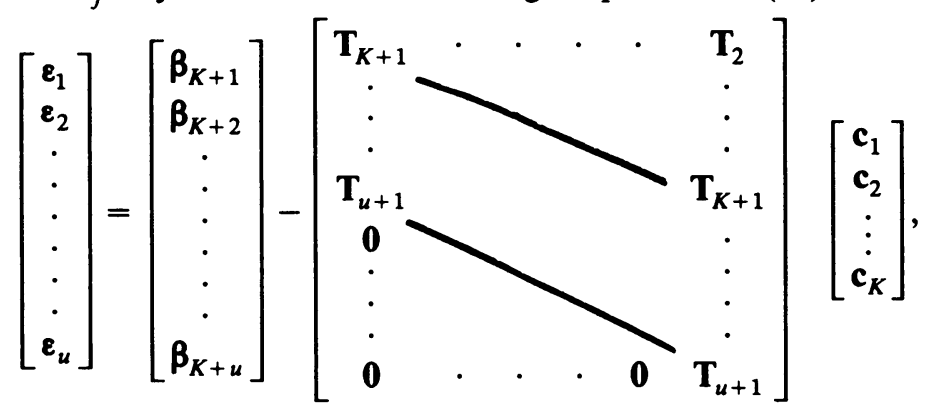

from which the $\varepsilon_{i}$ may be found. The matrix portion of (26) is written for the case that $u \geqslant K$. If $u<K$ this matrix should be replaced by

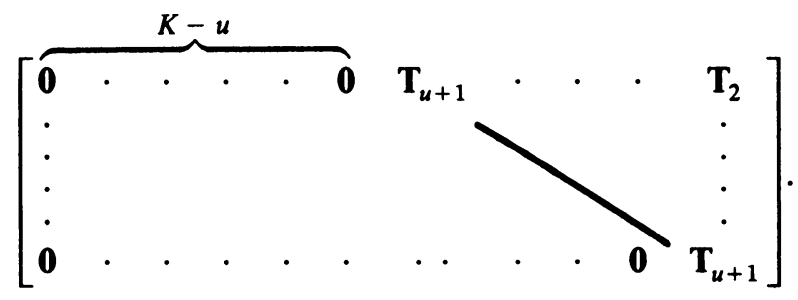


Evaluation of the $\mathbf{T}_{i} \cdot \mathbf{W}$ is easy to invert. It may be shown that

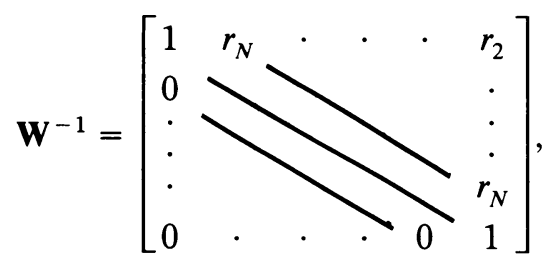

where

$$
r_{i}=q_{i}+\sum_{j=1}^{N-1} q_{j+1} r_{N+i-j}, \quad i=N, N-1, \ldots, 2 .
$$

By noting that

$$
\mathbf{W}^{-1} \mathbf{U}_{i}=\mathbf{U}_{i} \mathbf{W}^{-1}
$$

one may write (23) as

$$
\mathbf{T}_{i}=\mathbf{S}_{i}+\left(\mathbf{V} \mathbf{U}_{i}+\mathbf{U}_{i-1}\right) \mathbf{W}^{-1} .
$$

The first three $\mathbf{T}_{i}$ are now presented:

$$
N=1 \text { : }
$$

$$
\mathbf{T}_{i}=\alpha_{i 1}
$$

$$
N=2 \text { : }
$$

$$
\mathbf{T}_{i}=\left[\begin{array}{cc}
\alpha_{i 1} & \left(\alpha_{i-1,2}+q_{1} \alpha_{i 2}\right) \\
\alpha_{i 2} & \left(\alpha_{i 1}+q_{2} \alpha_{i 2}\right)
\end{array}\right]
$$

$N=3$ :

$$
\mathbf{T}_{i}=\left[\begin{array}{ccc}
\alpha_{i 1} & \left(\alpha_{i-1,3}+q_{1} \alpha_{i 3}\right) & \left(\alpha_{i-1,2}+q_{3} \alpha_{i-1,3}\right)+q_{1}\left(\alpha_{i 2}+q_{3} \alpha_{i 3}\right) \\
\alpha_{i 2} & \left(\alpha_{i 1}+q_{2} \alpha_{i 3}\right) & \left(\alpha_{i-1,3}+q_{1} \alpha_{i 3}\right)+q_{2}\left(\alpha_{i 2}+q_{3} \alpha_{i 3}\right) \\
\alpha_{i 3} & \left(\alpha_{i 2}+q_{3} \alpha_{i 3}\right) & \left(\alpha_{i 1}+q_{2} \alpha_{i 3}\right)+q_{3}\left(\alpha_{i 2}+q_{3} \alpha_{i 3}\right)
\end{array}\right] .
$$

By inspection of (31), (32), and (33), and through other considerations, a scheme for computing $\mathbf{T}_{i}$ which does not directly involve (30) may be obtained.

Let $t_{i j k}(j, k=1,2, \ldots, N)$ be the element in the $j$ th row and $k$ th column of $\mathbf{T}_{i}$. The first column of $\mathbf{T}_{i}$ is given by

$$
t_{i j 1}=\alpha_{i j}, \quad j=1,2, \ldots, N .
$$

For the ensuing columns $(k=2,3, \ldots, N)$ the first element is given by

$$
t_{i 1 k}=t_{i-1, N, k-1}+q_{1} t_{i N, k-1},
$$

while the remaining elements are obtained from

$$
t_{i j k}=t_{i, j-1, k-1}+q_{j} t_{i N, k-1}, \quad j=2,3, \ldots, N .
$$

Summary. Identify the polynomials $A(s), B(s)$, and $Q(s)$, as well as the numbers $K, N$ and $u$. Take the coefficients of $A(s)$ as the initial dividend, the numbers $q_{1}$, $q_{2}, \ldots, q_{N}$ as divisors, and use the extended version of Horner's scheme to find the coefficients of $\alpha_{1}(s), \alpha_{2}(s), \ldots, \alpha_{u}(s)$. Similarly, with the coefficients of $B(s)$ replacing those of $A(s)$ in Horner's scheme, the coefficients of $\beta_{1}(s), \beta_{2}(s), \ldots, \beta_{K+u}(s)$ may be found. By using (30) or from (34), (35), and (36), one may compute $\mathbf{T}_{1}$, 
$\mathbf{T}_{2}, \ldots, \mathbf{T}_{u+1}$. The vectors $\mathbf{c}_{1}, \mathbf{c}_{2}, \ldots, \mathbf{c}_{K}$ (and hence, the polynomials $C_{1}(s)$, $\left.C_{2}(s), \ldots, C_{K}(s)\right)$ may be gained from (25), while the vectors $\varepsilon_{1}, \varepsilon_{2}, \ldots, \varepsilon_{u}$ (and hence, the polynomials $\left.\varepsilon_{1}(s), \varepsilon_{2}(s), \ldots, \varepsilon_{u}(s)\right)$ come from (26). Finally, $E(s)$ is computed from (12). All the unknown quantities of (2) have been found.

Condition of $\mathbf{T}_{1}$. So far it has been tacitly assumed that $\mathbf{T}_{1}$ is nonsingular. If this is the case, (25) has a unqiue solution. Otherwise, (25) would either have no solution, or would have multiple solutions. We seek the conditions necessary to establish whether $\mathbf{T}_{1}$ is singular or not. The exposition is hampered by the fact that $\mathbf{T}_{1}$ depends upon $N$.

Let $z_{j}, j=1,2, \ldots, N$, be the zeros of $Q(\cdot)$. Assume that $\alpha_{1}\left(z_{j}\right)$ is an eigenvalue of $\mathbf{T}_{1}$. If the assumption is correct then the rows of $\mathbf{T}_{1}-\alpha_{1}\left(z_{j}\right) \mathbf{I}$ must be linearly dependent. In every case tested $(N=1,2,3$, and 4$)$, it was found that premultiplication of $\mathbf{T}_{1}-\alpha_{1}\left(z_{j}\right) \mathbf{I}$ by $\left[1, z_{j}, z_{j}^{2}, \ldots, z_{j}^{N-1}\right]$ resulted in the zero vector, indicating that the rows of $\mathbf{T}_{1}-\alpha_{1}\left(z_{j}\right) \mathbf{I}$ are linearly dependent. The details of the calculation are so regular that there is little reason to believe that the result is not general.

By setting $i=0$ in (5), it is seen that $A\left(z_{j}\right)=\alpha_{1}\left(z_{j}\right)$, and hence, the $A\left(z_{j}\right)$, $j=1,2, \ldots, N$, are the eigenvalues of $\mathbf{T}_{1}$. Since the determinant of a matrix is the product of its eigenvalues, it follows that

$$
\operatorname{det} \mathbf{T}_{1}=A\left(z_{1}\right) A\left(z_{2}\right) \cdots A\left(z_{N}\right),
$$

and hence, $\mathbf{T}_{1}$ is singular if and only if $A(\cdot)$ and $Q(\cdot)$ share a zero.

Example 1. This problem was suggested by Henrici [1, p. 556].

$$
\begin{aligned}
& A(s)=3 s^{3}-2 s^{2}+5 s+1, \\
& B(s)=4 s^{5}-2 s^{4}+2 s^{3}-s^{2}-8 s-9, \\
& Q(s)=(s-1), \quad K=3, \\
& N=1, \quad M=3, \quad m=5, \quad u=4 .
\end{aligned}
$$

By the standard version of Horner's scheme we compute

$$
\begin{aligned}
& \alpha_{11}=7, \quad \alpha_{21}=10, \quad \alpha_{31}=7, \quad \alpha_{41}=3, \quad \text { and } \\
& \beta_{11}=-14, \quad \beta_{21}=8, \quad \beta_{31}=33, \quad \beta_{41}=34, \quad \beta_{51}=18, \quad \beta_{61}=4 .
\end{aligned}
$$

From (25) and (31) we obtain

$$
\left[\begin{array}{rrr}
7 & 0 & 0 \\
10 & 7 & 0 \\
7 & 10 & 7
\end{array}\right]\left[\begin{array}{l}
c_{11} \\
c_{21} \\
c_{31}
\end{array}\right]=\left[\begin{array}{r}
-14 \\
8 \\
33
\end{array}\right]
$$

from which we get $c_{11}=-2, c_{21}=4, c_{31}=1$.

From (26)

$$
\left[\begin{array}{l}
\varepsilon_{11} \\
\varepsilon_{21} \\
\varepsilon_{31} \\
\varepsilon_{41}
\end{array}\right]=\left[\begin{array}{r}
34 \\
18 \\
4 \\
0
\end{array}\right]-\left[\begin{array}{lcc}
3 & 7 & 10 \\
0 & 3 & 7 \\
0 & 0 & 3 \\
0 & 0 & 0
\end{array}\right]\left[\begin{array}{r}
-2 \\
4 \\
1 \\
0
\end{array}\right]
$$

from which we find $\varepsilon_{11}=2, \varepsilon_{21}=-1, \varepsilon_{31}=1, \varepsilon_{41}=0$.

From (2) and (12) the decomposition becomes

$$
\frac{-2}{(s-1)^{3}}+\frac{4}{(s-1)^{2}}+\frac{1}{(s-1)}+\frac{(s-1)^{2}-(s-1)+2}{3 s^{3}-2 s^{2}+5 s+1} .
$$


Example 2. This problem was also taken from Henrici [1, p. 560].

$$
\begin{aligned}
& A(s)=3 s^{3}-2 s^{2}+5 s+1, \\
& B(s)=7 s^{4}-8 s^{3}+12 s^{2}-s+7, \\
& Q(s)=s^{2}+2 s+2, \quad K=1, \\
& N=2, \quad M=3, \quad m=4, \quad u=2 .
\end{aligned}
$$

The extended version of Horner's scheme yields

$$
\begin{aligned}
& \alpha_{11}=17, \quad \alpha_{12}=15 ; \quad \alpha_{21}=-8, \quad \alpha_{22}=3, \quad \text { and } \\
& \beta_{11}=-77, \quad \beta_{12}=-41 ; \quad \beta_{21}=28, \quad \beta_{22}=-36 ; \quad \beta_{31}=7 .
\end{aligned}
$$

From (25) and (32)

$$
\left[\begin{array}{ll}
17 & -30 \\
15 & -13
\end{array}\right]\left[\begin{array}{l}
c_{11} \\
c_{12}
\end{array}\right]=\left[\begin{array}{l}
-77 \\
-44
\end{array}\right]
$$

which yields $c_{11}=-1, c_{12}=2$. Then (26) gives

$$
\left[\begin{array}{l}
\varepsilon_{11} \\
\varepsilon_{12} \\
\varepsilon_{21} \\
\varepsilon_{22}
\end{array}\right]=\left[\begin{array}{r}
28 \\
-36 \\
7 \\
0
\end{array}\right]-\left[\begin{array}{rr}
-8 & 9 \\
3 & -14 \\
0 & 3 \\
0 & 0
\end{array}\right]\left[\begin{array}{r}
-1 \\
2
\end{array}\right] \text {, }
$$

from which $\varepsilon_{11}=2, \varepsilon_{12}=-5, \varepsilon_{21}=1, \varepsilon_{22}=0$. The decomposition is

$$
\frac{2 s-1}{s^{2}+2 s+2}+\frac{(1)\left(s^{2}+2 s+2\right)+(-5 s+2)}{3 s^{3}-2 s^{2}+5 s+1} \text {. }
$$

Operations Count. The sum of the number of multiplications and divisions required to implement (25) and (26) may be estimated. A number of assumptions are made. Among them are that $u=(M+1) / N$, and $v=(m+1) / N$. The enumeration computation is so complicated that only the final results are reported. The operations count for $(25)$ is

$N^{3}\left[\frac{3 \dot{u}-1}{6}\right]+N^{2}\left[K u+\frac{K}{2}+\frac{1}{2}+\dot{v} v-\frac{\dot{v} \dot{v}}{2}-\frac{\dot{v}}{2}-\frac{\dot{u}}{2}\right]+N\left[\frac{K}{2}+\dot{v}-\dot{u}-\frac{1}{3}\right]-\dot{v}$.

The values of $\dot{u}$ and $\dot{v}$ to be used are found in Figure 2 .

\begin{tabular}{l|l|l}
\hline & $1 \leqslant v<K$ & $K \leqslant v \leqslant K+u$ \\
\hline \multirow{2}{*}{$1 \leqslant u<K$} & $\dot{u}=u$ & $\dot{u}=u$ \\
& $\dot{v}=v$ & $\dot{v}=K$ \\
\hline \multirow{2}{*}{$K \leqslant u \leqslant \infty$} & $\dot{u}=K$ & $\dot{u}=K$ \\
& $\dot{v}=v$ & $\dot{v}=K$ \\
\hline
\end{tabular}

Figure 2

Proper values of $\dot{u}$ and $\dot{v}$.

The number of additional operations required for (26) is

$$
\frac{N^{2}}{2}\left[u^{2}+u+(\dot{v}-v)^{2}+\dot{v}-v\right]+N[\dot{u}-u]
$$

Henrici [2] gives the operations count for his Method C. In terms of the present notation that count is

$$
3 M N+\frac{1}{3} N^{3}+O\left(N^{2}\right) .
$$


The conditions under which this count was made correspond to those of the last row and last column of the table in Figure 2. The operations count for the method in this paper under those conditions is

$$
M[M+2 N+1]+\frac{1}{3} N^{3}+O\left(N^{2}\right),
$$

which is comparable to Henrici's result.

Department of Industrial and Systems Engineering

The University of Florida

Gainesville, Florida 32611

1. P. Henrici, Applied and Computational Complex Analysis, Vol. 1, Wiley, New York, 1974.

2. P. HENRICI, "An algorithm for the incomplete decomposition of a rational function into partial fractions,” Z. Angew. Math. Phys., v. 22, 1971, pp. 751-755. 